Provided for non-commercial research and education use. Not for reproduction, distribution or commercial use.

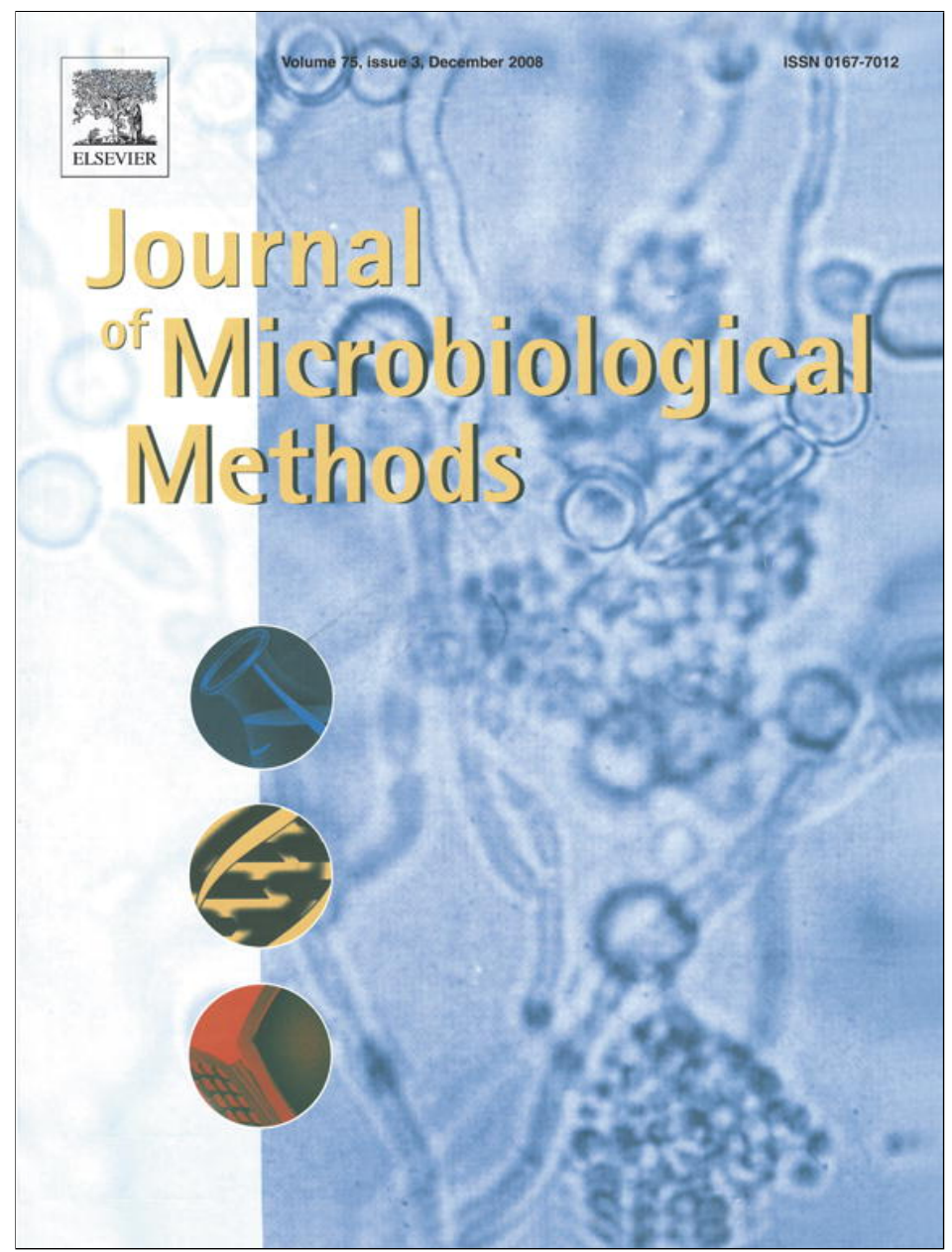

This article appeared in a journal published by Elsevier. The attached copy is furnished to the author for internal non-commercial research and education use, including for instruction at the authors institution and sharing with colleagues.

Other uses, including reproduction and distribution, or selling or licensing copies, or posting to personal, institutional or third party websites are prohibited.

In most cases authors are permitted to post their version of the article (e.g. in Word or Tex form) to their personal website or institutional repository. Authors requiring further information regarding Elsevier's archiving and manuscript policies are encouraged to visit:

http://www.elsevier.com/copyright 


\title{
Development of fluorescent in situ hybridisation for Cryptosporidium detection reveals zoonotic and anthroponotic transmission of sporadic cryptosporidiosis in Sydney
}

\author{
A. Alagappan ${ }^{\mathrm{a}, *}$, N.A. Tujula ${ }^{\mathrm{a}}$, M. Power ${ }^{\mathrm{b}}$, C.M. Ferguson ${ }^{\text {c,d }}$, P.L. Bergquist ${ }^{\mathrm{a}, \mathrm{e}}$, B.C. Ferrari ${ }^{\mathrm{a}, \mathrm{f}}$ \\ a Department of Chemistry and Biomolecular Sciences, Environmental Biotechnology CRC and Biotechnology Research Institute, Macquarie University, Sydney, NSW 2109, Australia \\ ${ }^{\mathrm{b}}$ Department of Biological Sciences, Macquarie University, Sydney, NSW 2109, Australia \\ ${ }^{\mathrm{c}}$ Ecowise Environmental, Fyshwick, ACT 2609, Australia \\ d Fenner School of Environment and Society, Australian National University, Canberra, ACT 0200, Australia \\ e Department of Molecular Medicine E Pathology, University of Auckland Medical School, Auckland, New Zealand \\ ${ }^{\mathrm{f}}$ School of Biotechnology and Biomolecular Sciences, University of New South Wales, Randwick, NSW 2052, Australia
}

\section{A R T I C L E I N F O}

\section{Article history:}

Received 16 July 2008

Received in revised form 19 August 2008

Accepted 21 August 2008

Available online 27 August 2008

\section{Keywords:}

18S rRNA

Anthroponotic

Cryptosporidium

FISH

Sporadic

Zoonotic

\begin{abstract}
A B S T R A C T
Cryptosporidium, is the most common non-viral cause of diarrhea worldwide. Of the 5 described species that contribute to the majority of human infections, C. parvum is of major interest due to its zoonotic potential. A species-specific fluorescence in situ hybridisation probe was designed to the variable region in the small subunit of the 18S rRNA of $C$. parvum and labeled with Cy3. Probe specificity was validated against a panel of 7 other Cryptosporidium spp. before it was applied to 33 human faecal samples positive for cryptosporidiosis which were obtained during the period from 2006-2007. Results were compared to PCR-RFLP targeting the 18S rDNA. FISH results revealed that 19 of the 33 isolates analysed were identified as $C$. parvum. Correlation of PCR-RFLP and FISH was statistically significant $(P<0.05)$, resulting in a calculated correlation coefficient of 0.994 . In this study, species identification by FISH and PCR-RFLP provided preliminary evidence to support both anthroponotic and zoonotic transmission of sporadic cases of cryptosporidiosis in the Sydney basin. In conclusion, FISH using a $C$. parvum-specific probe provided an alternative tool for accurate identification of zoonotic Cryptosporidium which will be applied in the future to both epidemiological and outbreak investigations.
\end{abstract}

(C) 2008 Elsevier B.V. All rights reserved.

\section{Introduction}

Cryptosporidium, the most common non-viral cause of diarrhea worldwide, is a protozoan parasite belonging to the Phylum Apicomplexa (Current and Garcia, 1991). Species belonging to the genus Cryptosporidium are the causative agents of a gastrointestinal infection, termed cryptosporidiosis (O'Donoghue, 1995). This diarrhoeal illness is self-limiting in immunocompetent patients but can be life-threatening in immunocompromised hosts, particularly in persons receiving immunosuppressive drugs and AIDS patients (Colford et al., 1997; Das, 1996). There is currently no effective therapy for the treatment or prevention of cryptosporidiosis in humans, despite intensive efforts over the past 20 years (Abrahamsen et al., 2004).

Although transmission routes include direct human to human contact, contaminated food and animal to human contact, a number of significant waterborne outbreaks have been reported worldwide, including Milwaukee (Mackenzie et al., 1994), Walkerton, Belfast and Clitheroe (Hrudey and Hrudey, 2004). While waterborne cryptosporidiosis outbreaks are occasionally detected, the source of the contamination is rarely identified, as this requires Cryptosporidium species

\footnotetext{
* Corresponding author. Tel.: +612 9385 2032; fax: +612 93851483.

E-mail address: aalagapp@bio.mq.edu.au (A. Alagappan).
}

identification and genotyping. Cryptosporidium species identification is not always part of the routine analyses performed by diagnostic pathology laboratories. Consequently, in Australia little information exists on the contribution of each species to disease burden.

Currently, there are 17 recognized species of Cryptosporidium, and thus far at least eight species have been identified in human infections; C. parvum, C. hominis, C. meleagridis, C. felis, C. canis, C. muris, C. suis and the Cryptosporidium cervine genotype (Xiao and Ryan, 2004). C. parvum and $C$. hominis are the primary species infecting humans (Morgan et al., 1998; Sulaiman et al., 1998; Xiao et al., 2002). C. hominis has an anthroponotic transmission cycle, while $C$. parvum has a predominantly zoonotic lifecycle, and is commonly found in a range of animals including cattle and sheep in addition to humans (Xiao and Ryan, 2004).

The epidemiological importance of human cryptosporidiosis has led to the development of new technologies for the identification of oocysts in clinical and environmental samples (Appelbee et al., 2005). Techniques for $C$. parvum identification such as immunofluorescence tend to overestimate pathogen load as the antibodies react with all species of Cryptosporidium (Bednarska et al., 2006). PCR-RFLP, the current industry gold standard for species identification, although sensitive, cannot assess viability of pathogens detected (Bednarska et al., 2006).

Fluorescence in situ hybridisation (FISH) meets two major challenges that is species-specific identification combined with simultaneous 
viability assessment. FISH utilizes fluorescently-labeled complementary DNA oligonucleotide probes that target specific sequences of cellular rRNA for direct identification of microorganisms (Amann et al., 1995). The rRNA is a naturally-amplified target for hybridisation probes due to its high copy number (Amann et al., 1990). FISH was first applied to detect Cryptosporidium oocysts in water samples which utilized a probe specific to a sequence of rRNA present in all Eukarya (Vesey et al., 1995). However, no FISH probes have been available which can differentiate C. parvum from C. hominis.

The purpose of the present study was to develop FISH using a species-specific probe that can be used as a reliable tool for the identification of zoonotic Cryptosporidium in human infections. The specificity of the C. parvum-specific FISH probe was investigated using viable oocysts of a range of Cryptosporidium species before it's validation on oocysts obtained from human faecal samples. Most importantly, the performance of FISH using C. parvum-specific probe against PCR-RFLP provided preliminary information on the contribution of disease burden in Sydney.

\section{Materials and methods}

\subsection{C. parvum probe design}

Complete 18S rRNA sequences of Cryptosporidium species were obtained from the GenBank database (http://www.ncbi.nlm.nih.gov/ genbank). The $18 \mathrm{~S}$ rRNA sequences were aligned using the program 'ClustalW' (Thompson et al., 1997) available through the Australian National Genomic Information Service (ANGIS website).

The sequences included in the alignment and their accession numbers are as follows: (C. muris Strain CMU03 AF093497, C. andersoni Strain bjcm AY954885, C. serpentis Strain CSP01 AF093499, C. baileyi Strain CBA01 AF093495, C. meleagridis Strain CMEL AF112574, C. parvum Strain K2 Kangaroo AF112570, C. wrairi Strain CWR AF115378, C. parvum Strain CPF AF112572, C. parvum Strain CPRM1 AF112569, C. hominis Strain HFL5 AF093492, C. hominis Strain HCNV4 AF093489, C. hominis Strain H7 AF108865, C. hominis Strain HFL2 AF093491, C. parvum Strain BOH6 AF093490, C. parvum Strain L16996, C. parvum Strain GCH1 AF093493, C. parvum Strain CPM1 AF112571, C. parvum Strain C1 AF108864, C. parvum Strain IOWA AF164102, C. parvum Strain UCP AF161857, C. parvum Strain HNJ-1 AB089290, C. parvum Strain MT AF161856, C. parvum Strain TAMU AF161858). Comparative analysis of the sequence alignment generated was carried out and a probe potentially specific for $C$. parvum was designed which targets one of the 4 variable regions in the 18S rRNA of C. parvum (Cpar677)

\subsection{Oocysts}

C. parvum oocysts were collected from naturally-infected neonatal calves from a dairy farm in Camden, Sydney. C. hominis and C. muris oocysts were sourced from our in-house Cryptosporidium isolate collection. Samples of cow faeces containing $C$. andersoni were kindly supplied by Wendi Smart from Lethbridge Research Centre, Canada. C. meleagridis, $C$. felis, $C$. cervine genotype and $C$. rabbit genotype (Robinson et al., in press) oocysts were from the national collection of Cryptosporidium oocysts at the UK Cryptosporidium Reference Unit, NPHS Microbiology Swansea, Singleton Hospital, Swansea, UK. All faecal and oocyst samples were stored at $4{ }^{\circ} \mathrm{C}$ without any preservative.

\subsubsection{FISH validation on uncharacterised oocysts}

Thirty three human faecal samples screened for Cryptosporidium and found to be positive by the Douglass Hanly Moir Pathology Laboratory in Sydney, Australia, were collected for this study. Faecal samples were processed within 2 weeks of sample collection. Samples were sieved through a series of meshes, centrifuged ( $3000 \mathrm{~g}, 10 \mathrm{~min}$ ) and resuspended in PBS $\left(4^{\circ} \mathrm{C}\right)$ prior to purification.

\subsection{Oocysts purification}

A modified sucrose floatation method was used to purify oocysts from cattle and human faecal samples (Truong and Ferrari, 2006). Oocyst samples were centrifuged (3000 g, $10 \mathrm{~min}$ ) and the resulting pellet resuspended in $20 \mathrm{ml}$ of Sucrose solution A (specific gravity 1.13-1.17), overlaid with $20 \mathrm{ml}$ of Sucrose solution B (specific gravity 1.08-1.10) and then overlaid with milliQ water. The gradient was centrifuged (3500 g, $30 \mathrm{~min}$ ) and oocysts collected between the solution B and the milliQ water layer. The oocysts were diluted in $>2$ vol of milliQ water and centrifuged ( $3000 \mathrm{~g}, 10 \mathrm{~min}$ ). The resulting pellet was resuspended in $10 \mathrm{ml}$ of $1 \%$ sodium bicarbonate $\left(\mathrm{NaHCO}_{3}\right)$. $10 \mathrm{ml}$ of diethyl ether was added, mixed thoroughly and centrifuged (3000 g, $10 \mathrm{~min}$ ). The ether layer was removed carefully before discarding the supernatant. The oocyst sample was allowed to air dry to remove ether residues. Oocysts were washed in $>2 \mathrm{vol}$ of phosphate-buffered saline (PBS, Oxoid) and centrifuged (3000 g, $10 \mathrm{~min}$ ). As a safety precaution, oocysts were irradiated by exposure to 1500 Ci from a ${ }^{60}$ Cobalt irradiation source for $24 \mathrm{~h}$ and stored in PBS at $4{ }^{\circ} \mathrm{C}$. Each purified oocyst sample was divided, with one portion for FISH and the remainder stored at $-20{ }^{\circ} \mathrm{C}$ for DNA extraction and further molecular analysis.

\subsection{Fluorescent in situ hybridisation using Cpar677 and EUK probes}

Cpar677 labelled with Cy3 and EUK labelled with Cy3 (Proligo, Australia) (Table 1) were used. FISH reactions were initially performed using a modified protocol previously described for Cryptosporidium (Dorsch and Veal, 2001).

\subsubsection{Fixation}

Briefly, a liquid suspension of oocysts was fixed in a solution of $50 \%$ PBS and $50 \%$ ethanol. They were further permeabilised by incubating at $80{ }^{\circ} \mathrm{C}$ for $20 \mathrm{~min}$. The fixed oocysts were dispensed onto $8 \mathrm{~mm}$ Isopore membrane filters (Millipore, Australia) by employing a vacuum manifold. Incubations were performed in plastic screwcapped lens containers (Bausch \& Lomb, inner diameter, $25 \mathrm{~mm}$ ) with the membrane filter carrying the oocysts placed on glass microfibre filters (Whatman $\mathrm{GF} / \mathrm{C}$, diameter $25 \mathrm{~mm}$ ).

\subsubsection{Hybridisation}

The filters were soaked in $300 \mu \mathrm{l}$ of pre-warmed $\left(46^{\circ} \mathrm{C}\right)$ hybridisation buffer ( $1 \mathrm{M}$ Tetramethyl ammonium chloride(TMAC), $50 \mathrm{mM}$ Tris [pH 8.0] containing $1 \mathrm{mM}$ EDTA [pH 8.0] and 0.1\% Sarkosyl). Hybridisation was carried out in hybridisation buffer containing oligonucleotide probes (EUK-Cy3 and Cpar677-Cy3 in separate reactions) at a final concentration of $3 \mathrm{mmol}^{-1}$. Oocysts were hybridised at $46{ }^{\circ} \mathrm{C}$ for $1 \mathrm{~h}$.

\subsubsection{Wash}

Membrane filters carrying oocysts were transferred to fresh filters soaked with $300 \mu \mathrm{l}$ of hybridisation buffer and washed twice at $48{ }^{\circ} \mathrm{C}$ for 30 min each.

Table 1

Oligonucleotide probes and fluorescence labels used in this study to identify and characterize Cryptosporidium

\begin{tabular}{llllll}
\hline $\begin{array}{l}\text { Probe } \\
\text { name }\end{array}$ & Sequence from $5^{\prime}$ to $3^{\prime}$ end & $\begin{array}{l}\text { Probe } \\
\text { position }\end{array}$ & Label & $\begin{array}{l}T_{\mathrm{m}} \\
\left({ }^{\circ} \mathrm{C}\right)\end{array}$ & Reference \\
\hline EUK & ACCAGACTTGCCCTCC & $502-516$ & Cy3 & 60 & $\begin{array}{l}\text { Amann et al. } \\
(1990)\end{array}$ \\
Cry1 & CGGTTATCCATGTAAGTAAAG & $138-160$ & FITC & 58 & $\begin{array}{l}\text { Vesey et al. } \\
(1998)\end{array}$ \\
Cpar677 & TCATATACTAAAATATATAGTAATAT & $677-702$ & Cy3 & 52 & This study \\
\hline
\end{tabular}

The probe target positions for Cry1 and Cpar677 are based on the 18S rRNA of C. parvum and EUK on the 16S rRNA of E. coli. 


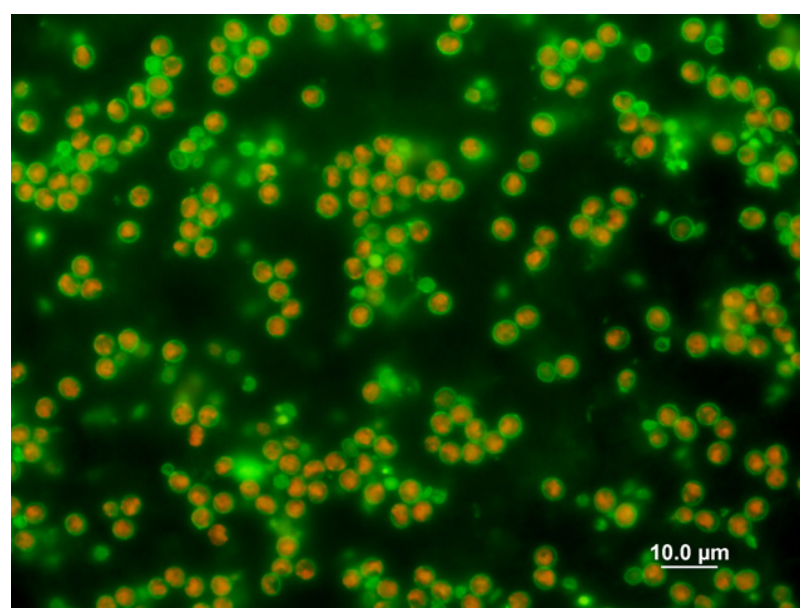

Fig. 1. FISH of C. parvum oocysts with Cy3-labelled 26-mer SSU rDNA oligonucleotide probe, Cpar677. Positive hybridisation was revealed by bright red fluorescence within oocysts. Oocysts were counterstained by immunolabelling (green) with CRY104-FITC. (For interpretation of the references to colour in this figure legend, the reader is referred to the web version of this article.)

\subsection{Counter-staining of oocysts}

The monoclonal antibody CRY104 conjugated to Fluorescein isothiocyanate (FITC) (BTF-North Ryde, Australia), specific to the wall of Cryptosporidium oocysts was used for confirmation of FISH-negative oocysts. The membranes were incubated in $100 \mu \mathrm{l}$ of monoclonal antibody buffer ( $2 \mathrm{mM}$ tetra sodium pyrophosphate (TSPP), 0.5\% (w/v) bovine serum albumin (BSA), $0.05 \%(\mathrm{v} / \mathrm{v})$ Tween $80, \mathrm{pH}$ adjusted to 8 using $1 \mathrm{M} \mathrm{HCl}$ ) containing FITC conjugated CRY104 (10 $\left.\mu \mathrm{g} \mathrm{ml}{ }^{-1}\right)$ for 3 min at RT and placed on a vacuum manifold to remove the staining liquid. The membranes were washed with $500 \mu \mathrm{l}$ of PBS and mounted onto microscope slides using $5 \mu \mathrm{l}$ Citifluor mountant media (Citifluor Ltd, UK) solution.

\subsection{Epi-fluorescence microscopy}

Epi-fluorescence microscopy was performed using an Axioskop 2 microscope fitted with $10 \times, 20 \times, 40 \times$ and a $100 \times$ oil immersion objectives (Carl Zeiss, Sydney, Australia). Excitation of cells was with a 100-W mercury vapour arc lamp combined with appropriate filters for excitation of FITC and Cy3. Recognition of C. parvum oocysts was accomplished at $100 \times$ magnification due to the combination of immunolabelling of the oocyst wall (green) for genus identification and bright hybridisation signals (red) from the species-specific C. parvum probe.

2.7. Evaluation of Cpar677 using human faecal samples positive for Cryptosporidium by FISH and PCR-RFLP

Oocysts from thirty-three human faecal samples were analysed by FISH and PCR-RFLP. Cryptosporidium oocysts from human faecal samples were identified to the species level using a PCR restriction fragment length polymorphism (PCR-RFLP) (Xiao et al., 2000) as a comparison against the FISH assay. DNA was extracted using a simple closed tube DNA extraction procedure based upon the use of a thermostable prepGem ${ }^{\mathrm{TM}}$ proteinase (Ferrari et al., 2007) for each purified oocyst sample from human faecal samples. DNA was eluted in $98 \mu \mathrm{l}$ of buffer prepGem ${ }^{\mathrm{TM}}$ (ZyGEM Corp Ltd, New Zealand) containing $10 \mathrm{mM}$ Tris (pH 7.5) and $1 \mathrm{mM}$ EDTA and stored at $-20{ }^{\circ} \mathrm{C}$.

A fragment of the 18S rRNA gene was amplified using a nested PCR procedure as previously described (Xiao et al., 2000). A 1325-bp PCR product was amplified first using primers 5'-TTCTAGAGCTAAT ACATGCG-3' and 5'-CCCATTTCCTTCGAAACAGGA-3'. A total of 35 cycles were carried out; each of these consisted of $94^{\circ} \mathrm{C}$ for $45 \mathrm{~s}, 55^{\circ} \mathrm{C}$ for $45 \mathrm{~s}$, and $72{ }^{\circ} \mathrm{C}$ for $1 \mathrm{~min}$. There was also an initial hot start at $94^{\circ} \mathrm{C}$ for $3 \mathrm{~min}$ and a final extension at $72{ }^{\circ} \mathrm{C}$ for $7 \mathrm{~min}$. A secondary 826- to 864-bp PCR product (depending on the isolate) was amplified from $1 \mu \mathrm{l}$ of the primary PCR mixture by using the primers, 5'-GGAAGGGTTGTATTTATTAGATAAAG-3' and 5'-AAGGAGTAAGGAACAACCTCCA-3'. The cycling conditions were identical to those used for the primary PCR. To differentiate between $C$. hominis and C. parvum, $20 \mu$ of each secondary PCR product was digested in a $50 \mu$ (total volume) reaction mixture containing $20 \mathrm{U}$ of $\operatorname{VspI}$ (Fermentas Life Sciences, Ontario) and $5 \mu \mathrm{l}$ of the appropriate restriction buffer for $1-2 \mathrm{~h}$, at $37{ }^{\circ} \mathrm{C}$ (Xiao et al., 2000). Digested products were separated on a $3.0 \%$ agarose gel and visualized by ethidium bromide staining. $C$. hominis and $C$. parvum were differentiated by specific banding profiles (Fig. 2). PCR products were purified using a QIAGEN PCR purification kit (QIAGEN, Germany) and sequenced using an Applied Biosystems 3130xl Genetic Analyser (Macquarie University, Sydney). Both strands of all PCR products of each ribotype were sequenced using the internal primers used in the $18 \mathrm{~S}$ nested PCR.

\subsection{Statistical analysis}

Data analysis and Pearson's correlation coefficient test at a significance level of 5\% were performed using Microsoft Excel 2003.

\section{Results}

\subsection{C. parvum probe design}

Based on phylogenetic comparisons in the 18S rRNA in Cryptosporidium spp., differences in oligonucleotide sequences within the

Table 2

Evaluation of C. parvum-specific probe - oocysts isolated from human faecal samples positive for Cryptosporidium by FISH and PCR-RFLP

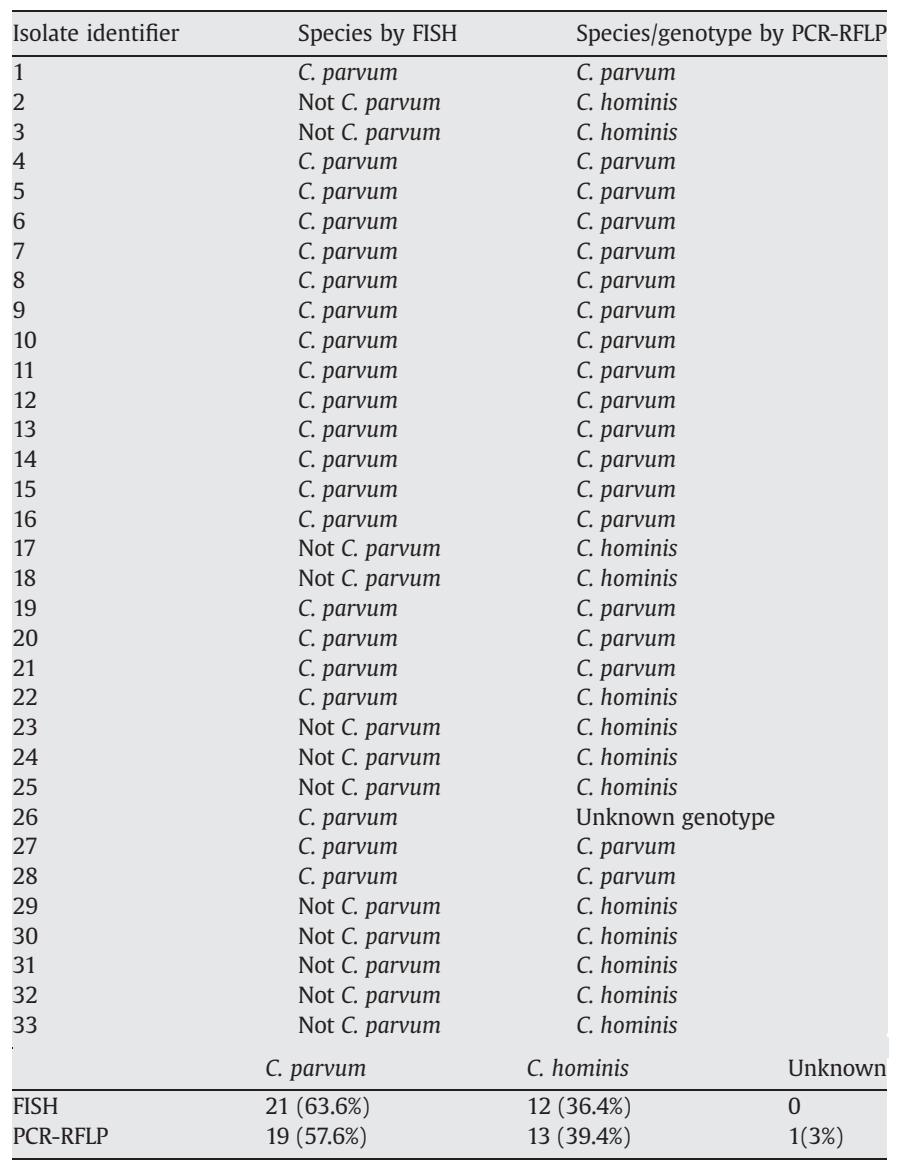


variable regions were predicted to be likely candidates for speciesspecific probes (Xiao et al., 1999). Comparative analysis of the sequence alignment generated by ClustalW (Thompson et al., 1997) led to the design of a probe potentially specific for C. parvum, Cpar677 (26-mer) with a predicted melting temperature $\left(T_{\mathrm{m}}\right)$ of $52^{\circ} \mathrm{C}$ (Table 1 ).

\subsection{Cpar677 probe specificity}

The specificity of the Cpar677-Cy3 probe was evaluated against a panel of 8 different Cryptosporidium species. C. parvum, C. hominis, C. andersoni, $C$. muris, $C$. meleagridis, $C$. felis, $C$. cervine genotype and C. rabbit genotype oocysts. The universal Eukaryotic probe EUK-Cy3 was first used to confirm sufficient rRNA for positive hybridisation to each of the species analysed. Oocysts were counterstained with CRY104-FITC and revealed bright green fluorescence of the oocyst wall when visualized with the green filter block on the epi-fluorescence microscope. Examination with the red filter block revealed positive red fluorescence due to hybridisation with EUK-Cy3. Evaluation of the Cpar677-Cy3 against each of the 8 species of oocysts revealed positive hybridisation (red fluorescence) only against C. parvum oocysts (Fig. 1). No red fluorescence was observed for the other species examined, indicative of specificity of this probe to the $C$. parvum sequence.

\subsection{Validation of Cpar677 against Cryptosporidium oocysts isolated from human faecal samples}

The specificity of Cpar677 probe for $C$. parvum oocyst detection was investigated further by using Cryptosporidium spp. oocysts of human origin. The probe was validated using oocysts purified from 33 human faecal samples (Table 2). Of the 33 isolates examined, 21 (63.6\%) tested positive with the Cpar677 probe, indicating the presence of $C$. parvum species. Of those exhibiting positive hybridisation, 20 isolates produced bright fluorescence signals, while a single isolate (22) yielded a weak fluorescent signal. The remaining 12 (36.4\%) isolates did not hybridise with the Cpar677 probe, indicating the absence of $C$. parvum in these samples.

3.4. Confirmation of oocyst species isolated from human faecal samples by PCR-RFLP

Confirmation using PCR-RFLP using VspI, revealed 19 of 33 isolates to be correctly identified as C. parvum (Fig. 2). With the exception of a single isolate (22), there was a strong correlation between C. parvumspecific FISH and RFLP (0.994). The RFLP profile of isolate 22 was indicative of the presence of $C$. hominis, while we observed mild crossreactivity with the $C$. parvum-specific probe in FISH.
Thirteen isolates were identified as $C$. hominis by PCR-RFLP analysis of the 18S rRNA gene. The RFLP pattern of one isolate 26 (unknown genotype), did not match the profiles of known Cryptosporidium spp. Sequencing of cloned PCR products showed it was most similar to C. hominis (AY204230) in both a nBLAST search and by using ClustalW alignment software. However, differences in the region of the $18 \mathrm{~S}$ rRNA were noted when comparing the unknown genotype sequence and the C. hominis (AY204230) sequence. The unknown genotype 18S rRNA differed from the $C$. hominis sequence with a T-A mutation at bp 694 , in the 689-699 basepair variable region. This single mutation in the variable region was a part of the sequence targeted by Cpar677 and hence the unknown genotype was genetically more similar to the C. parvum sequence than $C$. hominis and consequently hybridised with Cpar677 probe.

\section{Discussion}

Existing immunochromatographic and immunofluorescencebased assays, although sensitive and specific for the diagnosis of cryptosporidiosis in humans, do not provide species or genotype specific information. Rapid and accurate detection of Cryptosporidium species is crucial for the implementation of control measures and support strategies for epidemiologic investigations. While a simple yes/no morphology based test can detect the presence of Cryptosporidium spp in faecal samples, only molecular methods can guarantee identification at the species level. Identification at the species level is important for the determination of the source of infection (MorganRyan et al., 2002; Peng et al., 1997).

PCR has become increasingly common as a tool to detect Cryptosporidium DNA (Morgan et al., 1998). PCR allows for differentiation between different genotypes of the organism when followed by RFLP (Pedraza-Díaz et al., 2001; Spano et al., 1997). While this technique is sensitive and specific, the molecular analysis is time-consuming and requires expensive equipment and reagents. Direct identification of zoonotic Cryptosporidium species without the use of PCR based methods, would be of great value, during outbreak investigations. Simple tools that provide species-specific information would enable water utilities and public health authorities to better assess the potential human health risks associated with Cryptosporidium positive environmental samples.

Species-specific FISH is a reliable and robust alternative for the identification of $C$. parvum in a 3-hour timeframe. FISH is a simple technique that can be used in combination with currently existing immunofluorescence methods. Based on this study, it is clear that the $18 \mathrm{~S}$ rRNA can be used successfully for species-specific design of probes for FISH detection of Cryptosporidium spp. Species-specificity of the

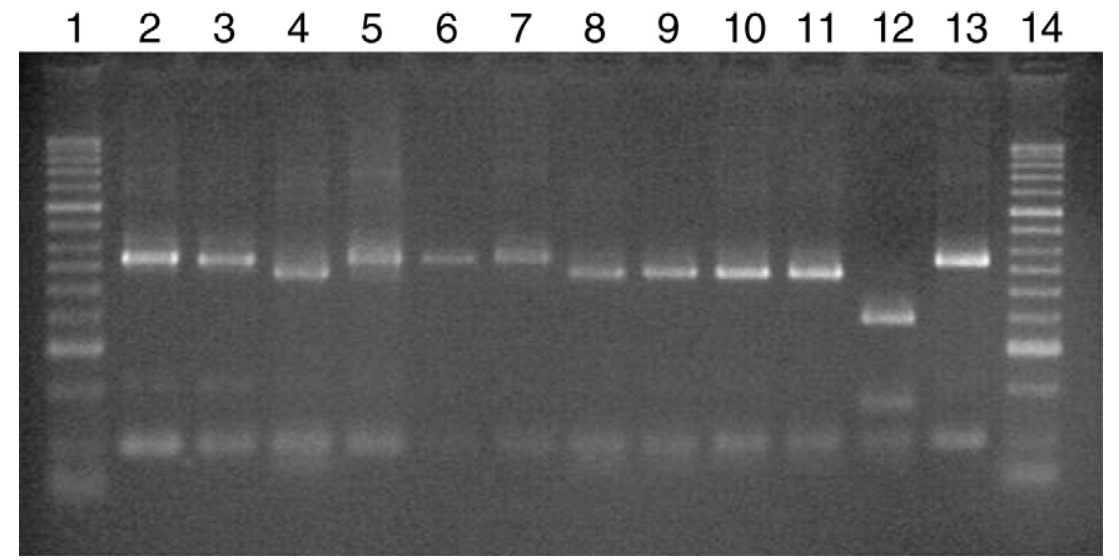

Fig. 2. Differentiation of Cryptosporidium by RFLP analysis of a PCR-amplified fragment of the $18 \mathrm{~S}$ rRNA gene. Secondary PCR products were digested with VspI. lanes 1 and 14,100 bp markers; lanes 2, 3, 5-7, 13, C. parvum; lanes 4, 8-11 C. hominis; lane 12, unknown genotype. 
C. parvum-specific probe was established following evaluation against a panel of 8 Cryptosporidium spp. Of the 8 species known to cause disease in humans, $C$. canis and $C$. suis could not be obtained for evaluation. However, analysis of the sequence of $18 \mathrm{~S}$ rRNA reveals that Cpar677 should not hybridise to these species (data not shown).

Extensive studies have been conducted over the last decade to understand the transmission of human cryptosporidiosis in developing countries with the help of genotyping and subtyping tools. Results from these studies have showed that $C$. parvum is responsible for slightly more infections than C. hominis in UK and other parts of Europe such as Northern Ireland, France, Switzerland, Portugal and New Zealand (McLauchlin et al., 2000; Chalmers et al., 2002; Leoni et al., 2006; Lowery et al., 2001; Coupe et al., 2005; Glaeser et al., 2004; Alves et al., 2003; Learmonth et al., 2004). In contrast, C. hominis is responsible for more infections than C. parvum in the United States, Canada, Australia and Japan (Peng et al., 1997; Morgan et al., 1998; Sulaiman et al., 1998; Ong et al., 1999; Ong et al., 2002; Xiao and Ryan, 2004). Interestingly, the results from our study provided preliminary evidence to support both anthroponotic and zoonotic transmission of sporadic cases of cryptosporidiosis in Eastern Australia. However, more comprehensive studies encompassing both rural and urban areas are needed to understand the transmission of sporadic cases of cryptosporidiosis in Australia.

When used in combination with a Cryptosporidium-specific antibody, species-specific FISH offers an alternative assay for rapid identification of zoonotic $C$. parvum in infectious samples. The total cost of one assay using this approach is approximately 5 -fold less than PCR-RFLP costs combined with a rapid turnaround time. FISH has the capacity to target a number of species simultaneously and in the future may be developed further for the identification of multiple species in a single assay using species-specific probes (Amann et al., 1990, 1995).

\section{Acknowledgements}

We thank Ann Cassidy of Douglass Hanly Moir Pathology, Sydney and Rogan Lee of ICPMR, Westmead Hospital, Sydney for the supply of Cryptosporidium-positive faecal samples; Quach Truong for technical support with faecal purifications; Debra Birch of Macquarie University for helping with microscopy. This work and Anitha Alagappan were supported by an Environmental Biotechnology CRC scholarship.

\section{References}

Abrahamsen, M.S., Templeton, T.J., Enomoto, S., Abrahante, J.E., Zhu, G., Lancto, C.A., 2004. Complete genome sequence of the apicomplexan, Cryptosporidium parvum. Science 304, 441-445

Alves, M., Xiao, L., Sulaiman, I., Lal, A.A., Matos, O., Antunes, F., 2003. Subgenotype analysis of Cryptosporidium isolates from humans, cattle and zoo ruminants in Portugal. J. Clin. Microbiol. 41, 2744-2747.

Amann, R.I., Binder, B.J., Olson, R.J., Chisholm, S.W., Devereux, R., Stahl, D.A., 1990. Combination of $16 \mathrm{~S}$ rRNA-targeted oligonucleotide probes with flow cytometry for analysing mixed microbial populations. Appl. Environ. Microbiol. 56, 1919-1925.

Amann, R., Ludwig, W., Schleifer, K., 1995. Phylogenetic identification and in situ detection of individual microbial cells without cultivation. Microbiol. Rev. 59, 143-169.

Appelbee, A.J., Thompson, R.C.A., Olson, M.E., 2005. Giardia and Cryptosporidium in mammalian wildlife - current status and future needs. Trends Parasitol. 21, 370-376.

Bednarska, M., Bajer, A., Sinski, E., Girouard, A.S., Tamang, L., Graczyk, T.K., 2006. Fluorescent in situ hybridization as a tool to retrospectively identify Cryptosporidium parvum and Giardia lamblia in samples from terrestrial mammalian wildlife. Parasitol. Res. 100, 455-460.

Chalmers, R.M., Elwin, K., Thomas, A.L., Joynson, D.H., 2002. Infection with unusual types of Cryptosporidium is not restricted to immunocompromised patients. J. Infect. Dis. 185, 270-271.

Colford, J., Tager, I., Hirozawa, A., Lemp, G., Aragon, T., Petersen, C., 1997. Cryptosporidiosis among patients infected with human immunodeficiency virus. Factors related to symptomatic infection and survival. Am. J. Epidemiol. 146, 807-816.

Coupe, S., Sarfati, C., Hamane, S., Derouin, F., 2005. Detection of Cryptosporidium and identification to the species level by nested PCR and restriction fragment length polymorphism. J. Clin. Microbiol. 43, 1017-1023.
Current, W.L., Garcia, L.S., 1991. Cryptosporidiosis. Clin. Microbiol. Rev. 4, 325-358.
Das, P., 1996. Cryptosporidium related diarrhoea. Indian J. Med. Res. 104, 86-95.

Dorsch, M., Veal, D., 2001. Oligonucleotide probes for specific detection of Giardia lamblia cysts by fluorescent in situ hybridization. J. Appl. Microbiol. 90, 836-842.

Ferrari, B., Power, M., Bergquist, P., 2007. Closed-tube DNA extraction using a thermostable proteinase is highly sensitive, capable of single parasite detection. Biotechnol. Lett. 29, 1831-1837.

Glaeser, C., Grimm, F., Mathis, A., Weber, R., Nadal, D., Deplazes, P., 2004. Detection and molecular characterization of Cryptosporidium spp. isolated from diarrheic children in Switzerland. Pediatr. Infect. Dis. J. 23, 359-361.

Hrudey, S.E., Hrudey, E.J., 2004. Safe Drinking Water - Lessons from Recent Outbreaks in Affluent Nations. IWA Publishing, London.

Learmonth, J.J., Ionas, G., Ebbett, K.A., Kwan, E.S., 2004. Genetic characterization and transmission cycles of Cryptosporidium species isolated from humans in New Zealand. Appl. Environ. Microbiol. 70, 3973-3978.

Leoni, F., Amar, R., Nichols, G., Pedraza-Diaz, S., McLauchlin, J., 2006. Genetic analysis of Cryptosporidium from 2414 humans with diarrhoea in England between 1985 and 2000. J. Med. Microbiol. 55, 703-707.

Lowery, C.J., Millar, B.C., Moore, J.E., 2001. Molecular genotyping of human cryptosporidiosis in Northern Ireland: epidemiological aspects and review. Ir J. Med. Sci. 170, 246-250.

Mackenzie, W., Hoxie, N., Proctor, M., Gradus, M., Blair, K., Peterson, D., 1994. A massive outbreak in Milwaukee of Cryptosporidium infection transmitted through the public water supply. N. Engl. J. Med. 331, 161-167.

McLauchlin, J., Amar, C., Pedraza-Diaz, S., Nichols, G.L., 2000. Molecular epidemiological analysis of Cryptosporidium spp. in the United Kingdom: results of genotyping Cryptosporidium spp. in 1,705 faecal samples from humans and 105 faecal samples from livestock animals. J. Clin. Microbiol. 38, 3984-3990.

Morgan, U.M., Pallant, L., Dwyer, B.W., Forbes, D.A., Rich, G., Thompson, R.C.A., 1998 Comparison of PCR and microscopy for detection of Cryptosporidium parvum in human fecal specimens: clinical trial. J. Clin. Microbiol. 36, 995-998.

Morgan-Ryan, U.M., Fall, A., Ward, L.A., Hijjawi, N., Sulaiman, I., Fayer, R., et al., 2002. Cryptosporidium hominis n. sp. (Apicomplexa: Cryptosporidiidae) from Homo sapiens. J. Eukaryot. Microbiol. 49, 433-440.

O'Donoghue, P., 1995. Cryptosporidium and cryptosporidiosis in man and animals. Int. J. Parasitol. 25, 139-195.

Ong, C.S., Eisler, D.L., Goh, S.H., 1999. Molecular epidemiology of cryptosporidiosis outbreaks and transmission in British Columbia, Canada. Am. J. Trop. Med. Hyg. 61, 63-69.

Ong, C.S., Eisler, D.L., Alikhani, A., Fung, V.W., Tomblin, J., Bowie, W.R., 2002. Novel Cryptosporidium genptypes in sporadic cryptosporidiosis cases: first report of human infections with a cervine genotype. Emerg. Infect. Dis. 8, 263-268.

Pedraza-Díaz, S.A.C., McLauchlin, J., Nichols, G.L., Cotton, K.M., Godwin, P., Iversen, A.M., 2001. Cryptosporidium meleagridis from humans: molecular analysis and description of affected patients. J. Infect. 42, 243-250.

Peng, M.M., Xiao, L., Freeman, A.R., Arrowood, M.J., Escalante, A.A., Weltman, A.C., 1997. Genetic polymorphism among Cryptosporidium parvum isolates: evidence of two distinct human transmission cycles. Emerg Infect Dis. 3, 567-573.

Robinson, G., Elwin, K., Chalmers, R.M., in press. Unusual Cryptosporidium genotypes in human cases of diarrhea. Emerg Infect Dis.

Spano, F., Putignani, L., McLauchlin, J., Casemore, D., Crisanti, A., 1997. PCR-RFLP analysis of the Cryptosporidium oocyst wall protein (COWP) gene discriminates between $C$. wrairi and $C$. parvum, and between $C$. parvum isolates of human and animal origin. FEMS Microbiol. Lett. 150, 209-217.

Sulaiman, I.M., Xiao, L., Yang, C., Escalante, L., Moore, A., Beard, C.B., 1998. Differentiating human from animal isolates of Cryptosporidium parvum. Emerg. Infect. Dis. 4, 681-685.

Thompson, J.D., Gibson, T.J., Plewniak, F., Jeanmougin, F., Higgins, D.J., 1997. The Clustal X windows interface: flexible strategies for multiple sequence alignment aided by quality analysis tools. Nucl. Acids Res. 25, 4876-4882.

Truong, Q., Ferrari, B., 2006. Quantitative and qualitative comparisons of Cryptosporidium faecal purification procedures for the isolation of oocysts suitable for proteomic analysis. Int. J. Parasitol. 36, 811-819.

Vesey, G., Ashbolt, N., Wallner, G., Dorsch, M., Williams, K., Veal, D., 1995. Assessing Cryptosporidium parvum oocyst viability with fluorescent in-situ hybridisation using ribosomal RNA probes and flow cytometry. Protozoan Parasites and Water, Cambridge: Royal Society of Chemistry, pp. 133-138.

Vesey, G., Ashbolt, N., Fricker, E.J., Deere, D., Williams, K.L., Veal, D.A., Dorsch, M., 1998. The use of a ribosomal RNA targeted oligonucleotide probe for fluorescent labelling of viable Cryptosporidium parvum oocysts. J. Appl. Microbiol. 85, 429-440.

Xiao, L., Ryan, U.M., 2004. Cryptosporidiosis: an update in molecular epidemiology. Curr. Opin. Infect. Dis. 17, 483-490.

Xiao, L., Morgan, U.M., Limor, J., Escalante, A., Arrowood, M., Shulaw, W., 1999. Genetic diversity within Cryptosporidium parvum and related Cryptosporidium Species. Appl. Environ. Microbiol. 65, 3386-3391.

Xiao, L., Singh, A., Limor, J., Graczyk, T., Gradus, S., Lal, A., 2000. Molecular characterisation of Cryptosporidium oocysts in samples of raw surface water and wastewater. Appl. Environ. Microbiol. 67, 1097-1101.

Xiao, L., Sulaiman, I.M., Ryan, U.M., Zhou, L., Atwill, E.R., Tischler, M.L., 2002. Host adaptation and host-parasite co-evolution in Cryptosporidium: implications for taxonomy and public health. Int. J. Parasitol. 32, 1773-1785. 